\title{
Fall Injuries and Depressive Symptoms Among Older Adults and the Mediating Effects of Social Participation - China, 2011-2018
}

\author{
Yalu Zhang'; Lei Zhang'; Xinhui Zhang'; Jingjing Sun'; Dongmin Wang, ${ }^{2, *}$; Gong Chen ${ }^{1, *}$
}

\begin{abstract}
Summary
What is already known on this topic?

Prior studies found that fall events were associated with a higher level of depressive symptoms and a lower level of social functioning and social participation. In addition, social participation has also been significantly associated with better conditions of depressive symptoms.
\end{abstract}

\section{What is added by this report?}

This article implemented the literature in three ways. First, it examined the mechanisms of social participation in the association between fall injuries and depressive symptoms among older adults in China. Second, it specified the fall-injured older adults group from those who merely experienced fall events. Third, it compared the results between rural and urban China and discussed policy implications for both groups.

What are the implications for public health practice?

Based on the findings of this study, future policies could consider boosting social participation at both the household and community level while taking into account the challenges of mobilities and social capabilities after fall injuries. Meanwhile, it is essential to accelerate the construction of aging-friendly communities to improve the accessibility of social participation and broaden social services to health management and monitoring.

Falls are the second leading cause of unintentional injury deaths worldwide. Older adults are one of the groups with the highest incidence of fall injuries. Globally, around $30 \%$ of socially active older adults (aged 65 and above) and about $50 \%$ of the oldest-old (aged 85 and above) experienced at least one fall in a year on average, with $4 \%-15 \%$ of these falls resulting in significant injuries (1). The situation is equally concerning in China. The annual rate of falls among older adults was about $11 \%-34 \%$, and $3.2 \%-17 \%$ of the falls were reported to need medical attention (2). Prior studies found that the incidence of falls was associated with depressive symptoms among older adults (3), which could then contribute again to repeated falls (4). However, few have investigated the role of social interventions in saving fall-injured older adults from experiencing depressive symptoms.

Using the Chinese Health and Retirement Longitudinal Study (CHARLS) 2011-2018 data (5), this study examined the potential mediating role of social participation in altering the effect of fall injuries on depressive symptoms. The results show that over $20.24 \%$ of older adults $(60+)$ experienced falls, among whom $46.77 \%$ needed at least 1 visit seeking medical treatment. Social participation accounted for partial effects of fall injuries on their depressive symptoms for rural older adults only, not their urban counterparts. For the first time, this study specified that the fallinjured older adults group from the group that merely had falls. Findings from this study emphasize the necessity of collective efforts from multiple levels to improve the social engagement of urban older adults who had fall injuries. Future studies could further specify what types of social participation would be more helpful in buffering the intervention effects.

This study used the longitudinal data from the China Health and Retirement Longitudinal Study 2011-2018. The baseline information of the data was collected in 2011 and was followed up in 2013, 2015, and 2018 ( 4 waves in total). The data were collected through stratified random sampling of about 10,000 households with permanent residents aged 45 years or older in 150 county-level units in 26 provincial-level administrative divisions (PLADs) in China, with a complete sample of about 17,000 people. This study pooled the interviewees who were 60 years old or above and had experienced falls between 2009 and 2018. The total final study sample was 7,980 people, including 3,728 people $(46.77 \%)$ who needed treatment for falls and 4,243 people $(53.23 \%)$ who needed no treatment for falls. In terms of the residential location, 2,968 people $(37.19 \%)$ in the analytical sample were in urban areas and 5,013 people (62.81\%) were in rural areas.

Depressive symptoms in this study were measured using the Center for Epidemiologic Studies Depression 
(CES-D) scale with ten questions to measure the feelings and behaviors of the interviewees one week before the interview date. Interviewees who missed three or more questions were removed. The mean scores of all answered questions were calculated for respondents who missed one to two questions and imputed for the missing questions for that respondent.

The baseline survey recorded whether the respondent had fallen in the two years prior to the interviews. The follow-up survey recorded whether the respondent had fallen since the last visit and how many severe falls required medical treatment. This study focused on older adults who had fall incidences between 2011 and 2018 and compared those who needed medical treatment and those who did not.

The CHARLS recorded respondents' interactions with family, friends, community, and social involvement in the month before the time of the interview. Respondents reported the frequency of each type of social activity they had attended. In this study, the frequency unit was unified as the day, and the frequencies were summed for each activity participated.

Furthermore, individual and household characteristics were controlled that may affect both the likelihood of fall injuries and the outcome variable of depressive symptoms. The individual characteristics include educational attainment, self-perceived health status, age, and marital status. The household characteristic includes the number of people living in the same household.

This study used mediation analysis to investigate the possible mechanism of the relationship between the independent variable (fall injuries), dependent variable (depressive symptoms), and the mediating variable (social participation). Baron and Kenny proposed a four-step process for establishing mediation (G). Following these steps, first, we investigated whether fall injury was associated with depressive symptoms. Second, we examined the association between fall injury and a potential mediator of social involvement to test the validity of the mediation analysis. Third, we examined the association between potential mediators of social participation and depressive symptoms. To further explore the possible mediating role of social involvement in the association between fall injury and depressive symptoms, we conducted a Sobel-Goodman mediation test using bootstrap replication (Figure 1). The mediation analysis provided evidence not only on whether fall injury had a direct effect and an indirect effect on depressive symptoms in older Chinese adults, but also indicated what proportion of the total effect was accounted for by the mediating factor of social involvement.

All the analyses were conducted within the national sample first and by urban and rural areas. The analytical software of Stata SE 15.0 (released in 2017 by StataCorp, LLC. in College Station, Texas, USA) was utilized to conduct the analyses stated above. The $\mathrm{R}$-squared value was used to measure to what extent the total variability was accounted for by this model. The significance level $(\alpha)$ of 0.05 was used as the cutoff point to indicate the probability of rejecting the null hypothesis in this study.

Two findings from the descriptive analysis should be noted. One is that the average percentage of fall injury incidence dramatically increased as ages increased. On average, nearly 5 people aged between 45 and 49 had fall injuries out of 100 peers. This number was as high as 9.6 among the older adults aged between 70 and 74 and reached up to 15.7 among the group between 85 and 89 years old (Figure 2). Another concerning

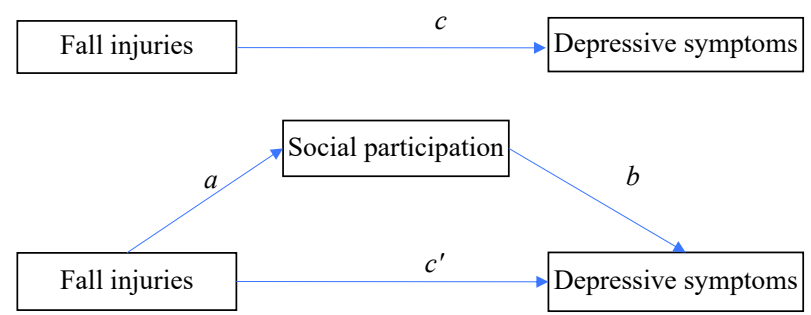

FIGURE 1. The demonstration of hypothesized mediated effects of social participation in the association between fall injuries and depressive symptoms.

Note: In the figure, path $c$ represents the total effect, path $c^{\prime}$ represents the direct effect, and the outcome of a followed by $b$ represents the average causal mediation effect (ACME).

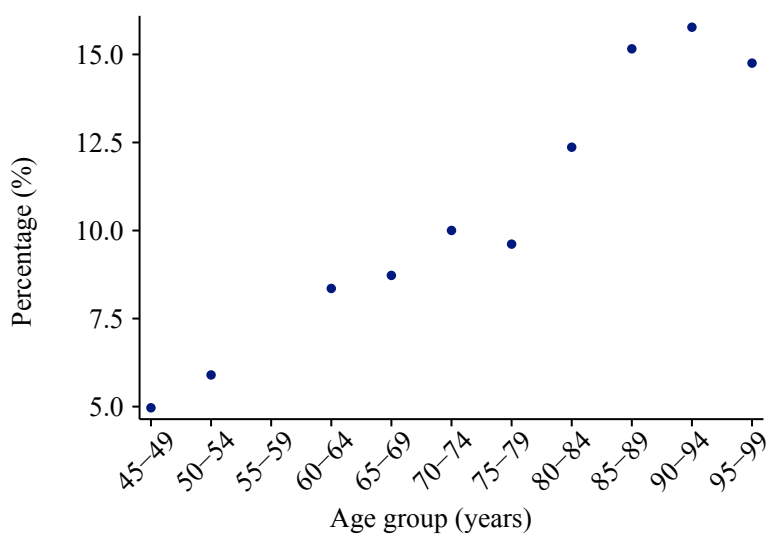

FIGURE 2. The average percentage of fall injury incidence by age groups for adults aged 45 years old and above, China, 2011-2018. 
finding was that compared to the urban older adults aged 60 and above, rural older adults were relatively more vulnerable as they were more likely to experience fall injuries, a higher level of depressive symptoms, worse health conditions, and less social participation (Table 1).

Using Baron and Kenny's mediating analytical strategies and the pooled cross-sectional data, the mediation analyses results showed that social participation accounts for a substantial proportion of fall injuries' effects on depressive symptoms among older adults in China (Table 2). More specifically, the likelihood of experiencing a higher level of depressive symptoms reduced from $7.63 \%$ to $6.73 \%$ for the fall-

TABLE 1. Descriptive statistics of falls, depressive symptoms, social participation, individual and household characteristics among older adults (aged 60 and above), China, 2011-2018.

\begin{tabular}{|c|c|c|c|c|c|c|c|c|c|}
\hline \multirow{2}{*}{ Variables } & \multicolumn{3}{|c|}{ National } & \multicolumn{3}{|c|}{ Rural } & \multicolumn{3}{|c|}{ Urban } \\
\hline & $\mathbf{N}$ & Mean & Std. Dev. & $\mathbf{N}$ & Mean & Std. Dev. & $\mathbf{N}$ & Mean & Std. Dev. \\
\hline Fall injury ratio (among people who had fall events) & 7,971 & 0.47 & 0.50 & 5,005 & 0.47 & 0.50 & 2,966 & 0.46 & 0.50 \\
\hline Fall injury ratio (among all older adults) & 39,395 & 0.09 & 0.29 & 23,844 & 0.10 & 0.30 & 15,551 & 0.09 & 0.28 \\
\hline Fall incidence & 39,424 & 0.20 & 0.40 & 23,868 & 0.21 & 0.41 & 15,556 & 0.19 & 0.39 \\
\hline Depressive symptoms & 32,637 & 1.54 & 0.80 & 19,569 & 1.63 & 0.84 & 13,068 & 1.41 & 0.71 \\
\hline Social participation & 46,561 & 0.62 & 0.95 & 27,064 & 0.54 & 0.82 & 19,497 & 0.74 & 1.10 \\
\hline Age & 46,561 & 69.48 & 7.64 & 27,064 & 69.48 & 7.62 & 19,497 & 69.49 & 7.68 \\
\hline Marital status & 46,516 & 0.78 & 0.41 & 27,058 & 0.78 & 0.42 & 19,458 & 0.80 & 0.40 \\
\hline Health condition & 40,931 & 2.92 & 0.98 & 24,623 & 2.85 & 0.98 & 16,308 & 3.02 & 0.96 \\
\hline People living in household & 46,521 & 1.49 & 1.86 & 27,055 & 1.64 & 1.98 & 19,466 & 1.27 & 1.65 \\
\hline
\end{tabular}

Note: The mean fall injury ratio (among people who had fall events) was obtained by dividing the number of people who experienced fall injuries by the number of people who experienced fall events. The mean fall injury ratio (among all the older adults) was obtained by dividing the number of fall injuries by the total analytical sample aged 60 and above. The mean fall incidence was obtained by dividing the number of fall events by the total analytical sample aged 60 and above.

Abbreviations: $\mathrm{N}=$ sample size; Std. Dev.=standard deviation.

TABLE 2. The mediating effects of social participation in the associations between fall injuries and depressive symptoms among older adults (aged 60 and above) in the national, urban, and rural sample, respectively, China, 2011-2018.

\begin{tabular}{|c|c|c|c|c|c|c|c|c|c|}
\hline \multirow[b]{2}{*}{ Variables } & \multicolumn{3}{|c|}{ National } & \multicolumn{3}{|c|}{ Urban } & \multicolumn{3}{|c|}{ Rural } \\
\hline & SocPart & Depress & $\begin{array}{c}\text { Mediated } \\
\text { effects }\end{array}$ & SocPart & Depress & $\begin{array}{c}\text { Mediated } \\
\text { effects }\end{array}$ & SocPart & Depress & $\begin{array}{c}\text { Mediated } \\
\text { effects }\end{array}$ \\
\hline \multirow{3}{*}{ SocPart } & & $b$ & & & $b$ & & & $b$ & \\
\hline & & $-0.0781^{*}$ & & & $-0.0633^{*}$ & & & $-0.0658^{*}$ & \\
\hline & & $(0.010)$ & & & $(0.013)$ & & & $(0.016)$ & \\
\hline \multirow{5}{*}{ Fall injuries } & $a$ & $c$ and $c^{\prime}$ & $c-c^{\prime}$ & $a$ & $c$ and $c^{\prime}$ & $c-c^{\prime}$ & $a$ & $c$ and $c^{\prime}$ & $c-c^{\prime}$ \\
\hline & $-0.1157^{*}$ & $0.0763^{*}$ & 0.0091 & $-0.2075^{\star}$ & 0.0583 & 0.0129 & $-0.0546^{\dagger}$ & $0.0827^{\S}$ & $0.0035^{\S}$ \\
\hline & $(0.026)$ & $(0.040)$ & $(0.004)$ & $(0.050)$ & $(0.064)$ & $(0.007)$ & $(0.027)$ & $(0.055)$ & $(0.003)$ \\
\hline & & $0.0673^{\S}$ & $11.85 \%$ & & 0.0454 & - & & $0.0792^{\dagger}$ & $4.18 \%$ \\
\hline & & $(0.040)$ & & & $(0.064)$ & & & $(0.056)$ & \\
\hline \multirow{2}{*}{$\begin{array}{l}\text { Individual characteristics } \\
\text { controlled for } \\
\text { Household characteristics } \\
\text { controlled for }\end{array}$} & Yes & Yes & Yes & Yes & Yes & Yes & Yes & Yes & Yes \\
\hline & Yes & Yes & Yes & Yes & Yes & Yes & Yes & Yes & Yes \\
\hline
\end{tabular}

Note: Standard error for path $a, b, c$ and $c$ ' in parentheses; Bootstrapped standard error for mediated effects in parentheses; The third line of each mediated effect indicates the proportion of total effect that is mediated; Depress stands for depressive symptoms; The individual characteristics include age, educational attainment, self-perceived health status, age, marital status; The household characteristic includes the number of people living in the same household; The mediated effect for the urban sample was not reported (noted as "-" in the table). Abbreviation: SocPart=social participation.

* $P<0.001$.

${ }^{\dagger} P<0.05$.

$\S P<0.01$. 
injured older adults when the older adults increased their social participation by $1 \% \quad(P<0.01)$. The mediated effect of social participation accounts for $11.85 \%$ [95\% Confidence Interval (CI): 7.66\%, $25.04 \%$ ] of the association.

Given that there exist significant variations between rural and urban older adults in terms of the frequency and possibility of experiencing fall injuries and levels of depressive symptoms, social participation, and individual and household characteristics, this study conducted separate mediating analyses for urban and rural older adults. The findings showed that the mechanisms varied across the urban and rural areas as hypothesized. For rural older adults, the probability of experiencing a higher level of depressive symptoms reduced by $3.5 \%$ for the fall-injured older adults when the older adults boosted their social participation by $1 \%$. The mediated effect size of social participation accounts for $4.18 \%$ (95\% CI: $2.53 \%, 11.98 \%$; $P<0.01)$. However, for urban older adults, the results do not show that social participation altered the likelihood of having depressive symptoms among fallinjured older adults.

\section{DISCUSSION}

Previous studies have focused on the impact of fall events on physical and mental health status and social functioning among older adults and findings have shown that fall events are positively associated with depressive symptoms and negatively associated with social functioning and social participation (7-10). Additionally, social participation is found to significantly alleviate depressive symptoms (11). A few scholars also examined the impacts of fall events on social behaviors, living arrangements, and depressive symptoms (2,12-13). Nevertheless, limited studies have differentiated between the concepts of fall events and fall injuries caused by fall events that need medical treatment. In addition to the physical damages, fall injuries, as specific outcomes of the fall event, could also produce physiological damage and warrants further investigation regarding the significant negative impact of older adults' mental health such as depressive symptoms and fears of falling.

Therefore, this study makes contributions in three aspects. First, it investigated the mechanism of social involvement as an essential mediator for the association between fall injuries and depressive symptoms among older adults. Second, it focused on the older adults who experienced fall injuries to provide evidence for future intervention implementation and evaluation for the more severe outcomes of fall events. Third, it tested the mediating effects for both urban and rural older adults to provide more specific evidence for future policymaking and service provision.

This study was subject to at least two limitations. First is that for the purpose of having a larger sample size, this study used the pooled cross-sectional data, which could not examine the long-term mediating effects of social participation. Second is that due to the data limitations, this study did not specify the types of social participation, such as family interactions, community activity participation, social engagement, etc., which could vary the outcomes.

The findings from this study suggest that improving rural older adults' social participation could be an intervention to prevent the fall injured older adults from higher levels of depressive symptoms. Future policies could consider boosting social participation at both the household and community level, removing barriers for older adults to access online social participation, while taking into account the challenges of mobilities and social capabilities after the fall injuries. Meanwhile, this study provides evidence that it is essential to accelerate the construction of agingfriendly communities to improve accessibility of social participation and broaden the social services such as health management and chronic diseases monitoring.

Based on the findings that social participation does not function as a mediator to the association between fall injuries and depressive symptoms for Chinese urban older adults, future research could consider examining other potential mechanisms for this group of population. Additionally, future studies could decompose the types of social participation into family interactions, community-based activities, internet social participation, and social volunteering activities.

Acknowledgments: The China Health and Retirement Longitudinal Study (CHARLS) team.

Conflicts of interest: No conflicts of interest.

Funding: Funded by the China's National Key Research and Development Program (2018YFC 2000603).

\section{doi: $10.46234 / \mathrm{ccdcw} 2021.207$ \\ \# Corresponding authors: Dongmin Wang, dongmin_wang@pku. edu.cn; Gong Chen, chengong@pku.edu.cn.}

\footnotetext{
${ }^{1}$ Institute of Population Research, Peking University, Beijing, China; 2 Department of Physical Education, Peking University, Beijing, China.
}

Submitted: September 12, 2021; Accepted: September 22, 2021 


\section{REFERENCES}

1. World Health Organization. World report on ageing and health. 2015. https://apps.who.int/iris/handle/10665/186463. [2021-9-12].

2. World Health Organization. China country assessment report on ageing and health. 2015. https://www.who.int/publications/i/item/978924150 9312. [2021-9-12].

3. Atlas A, Kerse N, Rolleston A, Teh R, Bacon C. Falls and depression in octogenarians-life and living in advanced age: a cohort study in New Zealand. J Prim Health Care 2017;9(4):311 - 5. http://dx.doi.org/10. 1071/HC17012.

4. Hoffman GJ, Hays RD, Wallace SP, Shapiro MF, Ettner SL. Depressive symptomatology and fall risk among community-dwelling older adults. Soc Sci Med 2017;178:206 - 13. http://dx.doi.org/10. 1016/j.socscimed.2017.02.020.

5. Zhao YH, Hu YS, Smith JP, Strauss J, Yang GH. Cohort profile: the China health and retirement longitudinal study (CHARLS). Int J Epidemiol 2014;43(1):61 - 8. http://dx.doi.org/10.1093/ije/dys203.

6. Baron RM, Kenny DA. The moderator-mediator variable distinction in social psychological research: conceptual, strategic, and statistical considerations. J Pers Soc Psychol 1986;51(6):1173 - 82. http://dx. doi.org/10.1037/0022-3514.51.6.1173.

7. Ganz DA, Latham NK. Prevention of falls in community-dwelling older adults. N Engl J Med 2020;382(8):734 - 43. http://dx.doi.org/
10.1056/NEJMcp1903252.

8. Hughes CC, Kneebone II, Jones F, Brady B. A theoretical and empirical review of psychological factors associated with falls-related psychological concerns in community-dwelling older people. Int Psychogeriatr 2015; 27(7):1071 - 87. http://dx.doi.org/10.1017/S1041610214002701.

9. van der Meulen E, Zijlstra GAR, Ambergen T, Kempen GIJM. Effect of fall-related concerns on physical, mental, and social function in community-dwelling older adults: a prospective cohort study. J Am Geriatr Soc 2014;62(12):2333 - 8. http://dx.doi.org/10.1111/jgs.13083.

10. Zhou XF, Wang JL. Correlation between fear of tumble and anxietydepression of 480 community elderly people. Chin J Convalescent Med 2010;19(2):189 - 90. http://dx.doi.org/10.3969/j.issn.1005-619X. 2010.02.078. (In Chinese).

11. Quach LT, Ward RE, Pedersen MM, Leveille SG, Grande L, Gagnon $\mathrm{DR}$, et al. The association between social engagement, mild cognitive impairment, and falls among older primary care patients. Arch Phys Med Rehabil 2019;100(8):1499 - 505. http://dx.doi.org/10.1016/ j.apmr.2019.01.020.

12. Durgun H, Turan N, Kaya H. Relationship between fall behavior and quality of life of elderly individuals. Psychol Health Med 2021;1 - 8 . http://dx.doi.org/10.1080/13548506.2021.1883686.

13. Bu FF, Abell J, Zaninotto P, Fancourt D. A longitudinal analysis of loneliness, social isolation and falls amongst older people in England. Sci Rep 2018;10(1):20064. http://dx.doi.org/10.1038/s41598-02077104-z. 